\title{
MESENCHYMAL STEM CELLS FOR MANAGEMENT OF RHEUMATOID ARTHRITIS - IMMUNE MODULATION, REPAIR OR BOTH?
}

\author{
Sharon Ansboro, Anke J. Roelofs, Cosimo De Bari
}

Arthritis \& Regenerative Medicine Laboratory, Aberdeen Centre for Arthritis and Musculoskeletal Health, Institute of Medical Sciences, University of Aberdeen, Aberdeen AB25 2ZD, United Kingdom

Corresponding author: Professor Cosimo De Bari, MD PhD FRCP, Institute of Medical Sciences, University of Aberdeen, Foresterhill, Aberdeen AB25 2ZD, UK. Tel: +44-1224437477, Fax: +44-1224-437348, E-mail: c.debari@abdn.ac.uk. 


\section{Purpose of Review}

Mesenchymal stromal/stem cells (MSCs) have potent anti-inflammatory and immunomodulatory properties, in addition to their ability to form cartilage and bone. The purpose of this review is to highlight recent developments and current knowledge gaps in our understanding of the protective effects of MSCs against inflammatory arthritis, and to discuss their clinical exploitation for the treatment of rheumatoid arthritis (RA).

\section{Recent Findings}

The weight of evidence for protective mechanisms of exogenously administered MSCs is on immunomodulatory effects, including inhibition of dendritic cell maturation, polarisation of macrophages to an anti-inflammatory phenotype, and activation of regulatory $\mathrm{T}$ cells, thereby dampening inflammation and preventing joint damage. Evidence for direct effects on tissue repair is scant. Recent studies have identified MSC subsets in vivo and an important question is whether MSCs in their native tissues have similar immunoregulatory functions. Recent proof-of-concept clinical studies have shown a satisfactory safety profile of allogeneic MSC therapy in RA patients with promising trends for clinical efficacy.

\section{Summary}

Allogeneic MSCs could be effective in RA. Larger, multicentre clinical studies are needed to provide robust evidence, and MSC treatment at early stages of RA should be explored to "reset" the immune system.

Keywords: Mesenchymal Stem/Stromal Cells, Rheumatoid Arthritis, Immunomodulation, Cartilage Repair 


\section{Introduction}

Rheumatoid arthritis (RA) is a chronic autoimmune inflammatory joint disease. The cellular effectors include both innate and adaptive immune cells. In addition, fibroblast-like synoviocytes (FLS) adopt an aggressive and invasive phenotype which contributes to joint damage, and increased osteoclast activity mediates excessive bone resorption. Current treatment strategies mainly aim to suppress autoimmune inflammation using diseasemodifying anti-rheumatic drugs and biologics. While the success of immune modulation and cytokine inhibition is considerable, $\sim 30 \%$ of patients in trials do not respond satisfactorily to treatments[1,2]. Even when clinical remission is achieved, cartilage and bone damage may be established or continue to progress. A challenging goal is to induce remission via permanent immune tolerance, protect against structural damage, and repair existing damage.

Mesenchymal stromal/stem cells (MSCs) have immunomodulatory and tissue-repair properties, and their use for management of RA is being explored. The original definition of human MSCs is based on in vitro properties of isolated and culture-expanded plastic-adherent cells, namely tri-lineage differentiation to osteoblasts, chondrocytes and adipocytes, and expression of CD73, CD90 and CD105, but not haematopoietic or endothelial markers[3]. MSCs have been isolated from several connective tissues, including bone marrow[4], synovium[5,6], periosteum[7,8], adipose tissue[9], and umbilical cord[10] (Figure 1).

MSCs can regulate inflammation via an array of mechanisms, involving both the adaptive and innate immune response. These include inhibition of $\mathrm{T}$ cell proliferation and function, induction of $\mathrm{CD}^{+} \mathrm{CD} 25^{+} \mathrm{FoxP}^{+}$regulatory $\mathrm{T}$ cells (Tregs), suppression of B-cell proliferation, differentiation, and immunoglobulin production, suppression of dendritic cell maturation, promotion of macrophage polarisation towards an anti-inflammatory phenotype, and suppression of NK cells (for review see[11]). Immunomodulation by MSCs is mediated via both direct cell-cell contact and secretion of soluble factors such as prostaglandin E2 (PGE2), indoleamine 2,3-dioxygenase (IDO), nitric oxide (NO) and Interleukin-10 (IL-10), released in response to stimulation by IFN- $\gamma$ from activated immune cells[11]. All these mechanisms could contribute to resolution of inflammation in RA.

This review will examine MSCs as a possible therapy for RA by critically assessing recent literature on the mechanisms by which MSCs modulate the immune system and promote repair. 


\section{Insights into MSC immunomodulatory mechanisms in RA}

Preclinical studies have shown beneficial effects of MSC therapy in models of RA-like inflammatory arthritis[12]. One of the first studies to demonstrate protective effects of intraperitoneally injected mouse MSCs against joint destruction in collagen-induced arthritis (CIA) failed to detect administered MSCs in the joints, suggesting that prevention of joint damage resulted predominantly from a dampening down of the immune system[13].

Modulation of $\mathrm{T}$ cell function, including suppression of $\mathrm{T}$ cell proliferation and activation of Tregs, has been implicated[13,14,15], and recent studies have provided additional insights into the immunomodulatory effects of MSCs in inflammatory arthritis. Lopez-Santalla et al.[16*] showed that intravenous administration of human adipose-MSCs (Ad-MSCs) in CIA mice decreased GM-CSF-expressing CD4+ $\mathrm{T}$ cells, key effector cells in RA pathophysiology[17], in blood and spleen. Regulatory T cells, including classical Tregs and IL10 ${ }^{+}$IL17 ${ }^{-} \mathrm{CD} 4^{+} \operatorname{Tr} 1$ cells, were decreased in spleen and increased in draining lymph nodes, suggestive of their mobilisation towards inflamed tissues. In addition, an increased proportion of Th17 cells expressed the anti-inflammatory cytokine IL-10[16*], suggesting that the previously reported induction of a regulatory phenotype in Th17 cells by MSCs[18,19] also occurs in vivo in the context of CIA. Indeed, this was confirmed in a study by Luz-Crawford et al. that further showed this to be dependent on glucocorticoid-induced leucine zipper (GILZ), which inhibits the pro-inflammatory transcription factors NF- $x \mathrm{~B}$ and activator protein 1 (AP-1), in MSCs[20**]. Downregulation of NF-xB signalling may also occur via decreased expression of microRNA (miR)-548e and a resulting de-repression of IкB translation[21*]. Intraperitoneal injection of MSCs in conjunction with a miR-548e-encoding adeno-associated virus prevented the beneficial effects of MSC transplantation on CIA, while intraperitoneal injection of antisense-miR-548e alone showed improved arthritis outcome, although the main cell type targeted was not clear from the evidence provided[21*]. Follicular helper T (Tfh) cells, which provide proliferative signals to B cells in secondary lymphoid tissues[22], have also been implicated in the immunosuppressive effects of MSCs. Umbilical cord (UC) MSCs inhibited Tfh cell differentiation in vitro, and intravenous administration of human UC-MSCs in mice after onset of CIA decreased the number of Tfh cells in the spleen, and suppressed their capacity to support B lymphocyte differentiation in an ex vivo co-culture assay[23**]. Inhibitory effects of MSCs on B cells were recently shown 
to be dependent on interactions between MSCs and T cells[24*], and effects on Tfh cells could thus mediate the indirect suppressive effects of MSCs on B cells.

Strategies to augment the immunomodulatory potency of MSCs have been explored to enhance therapeutic efficacy. For example, co-administration of MSCs and Tr1 cells was more effective in reducing inflammation, pannus formation and cartilage erosion in the CIA model compared to single cell therapy, possibly through increased IDO expression in MSCs induced by IFN- $\beta$ and IL-10 produced by the Tr1 cells[25*]. Another strategy involved engineering of MSCs with microparticles loaded with the glucocorticoid budesonide. Such MSCs exhibited enhanced IDO activity compared to budesonide-preconditioned and naïve MSCs, resulting in improved in vitro immunosuppression[26].

Protective effects of MSCs against excessive osteoclast-mediated bone resorption, resulting in local and systemic bone loss, are likely mediated via suppression of inflammatory cytokines that promote osteoclastogenesis[27], and independently, via boosting of Tregs[28,29]. Direct inhibitory effects of MSCs on osteoclastogenesis via production of the RANKL decoy receptor osteoprotegerin[30], or through CD200/CD200R-dependent inhibitory interactions with osteoclast precursors[31], have also been suggested. In addition, a recent study reported that prevention of local and systemic bone loss by administration of syngeneic Ad-MSC to CIA mice was associated with a decrease in CD11 $\mathrm{b}^{+} \mathrm{c}-\mathrm{fms}^{+}$osteoclast precursors in bone marrow[32*], though the mechanisms remain to be elucidated.

Of note, several studies have failed to demonstrate an improvement in experimental CIA with MSC treatment, with some even reporting a worse outcome[12]. In a comprehensive study, Schurgers et al.[33] did not detect any benefit from MSC therapy in CIA, using both intravenous and intraperitoneal routes to administer either syngeneic or allogeneic MSCs. In contrast, injection of Tregs before or after disease onset let to a dramatic improvement of arthritis[33]. Contradictory results may arise from variables including source of MSCs, tissue of origin, MSC culture conditions, timing of treatment, number of cells injected, route of injection and treatment regime[10]. Although MSCs have low immunogenicity and display low levels of MHC I and absence of MHC II and co-stimulatory molecules, culture expansion and differentiation of MSCs into mature cell types can increase the expression of MHC class I and II molecules[34], and allogeneic MSCs may elicit both a humoral and cellular response in vivo[35,36,37]. Hence, these cells may not be completely immune privileged. Donor- 
variability is also likely a confounding factor and many studies fail to demonstrate whether findings are reproducible using MSCs from different human donors.

\section{The influence of tissue source on MSC potency}

MSC from several tissues possess immunomodulatory properties, adding to the MSC armamentarium, but also raising critical questions regarding their equivalence vs. diversity in potency and clinical effectiveness. The potency of MSCs appears to be dependent on, among other factors, the ontogenetic pathways through embryonic tissue formation and the adult tissues in which MSCs reside postnatally. For instance, human MSCs from synovium display greater chondrogenic potency in vitro when compared with MSCs from bone marrow, periosteum and adipose tissue[38], while they are inferior to periosteal MSCs in boneforming potency in vivo[39]. Similarly, tissue source seems to affect the immunosuppressive effects of MSCs. For example, Ad-MSCs were found to have a greater immunosuppressive capacity on T cells and monocytes in comparison to bone marrow (BM) MSCs[40].

In addition to the plethora of "finite" adult MSCs, MSCs could be derived from embryonic stem cells, recognised as a potential "infinite" and more easily standardisable source of MSCs. It was recently shown that intraperitoneal administration of MSCs derived from human embryonic stem cells can ameliorate CIA when administered after disease onset. Injected MSCs were found to home to draining lymph nodes and to upregulate IDO expression by the host[ $41 *]$.

\section{Identification of MSC subsets in vivo}

Recent advances have been made in our understanding of the identity and functions of MSCs in vivo in their native tissues, mainly in bone marrow. Subpopulations of MSCs in mouse bone marrow are marked by Pdgfra and Sca1[42], leptin receptor[43,44], Nestin[45], or Gremlin-1[46**], with varying degrees of overlap. MSCs have also been identified in mouse synovium[47]. It is becoming clear that different MSC subsets variably contribute to the formation of mesenchymal tissues during development and growth, adult tissue turnover, and following injury. In human bone marrow, MSCs are marked by LNGFR (CD271)[48,49] and CD146[50,51], with the latter shown to be a perivascular subset of LNGFR-expressing cells[52]. Pdgfra and CD51 mark a subset of CD146 ${ }^{+}$MSCs that are Nestin ${ }^{+}$in both adult 
mouse and foetal human bone marrow[53], although Li et al. reported MSCs in adult human bone marrow to be enriched within the Pdgfra ${ }^{\text {low/neg }}$ fraction of $\mathrm{LNGFR}^{+}$cells[54].

Discovery of specific markers will allow isolation of defined MSC populations using standardised protocols, which will aid consistency in research and translation to clinic. In this regard, an important question is whether MSC subpopulations show varying immunomodulatory potency. A recent study[55*] reported that intra-articular injection of CD146 ${ }^{+}$UC-MSCs but not CD146- cells ameliorated CIA in mice. However, sorting for CD146 expression in this study was performed on culture-expanded cells. Tormin et al.[52] showed that CD146 expression by freshly sorted CD146 ${ }^{\text {neg/low }}$ LNGFR $^{+}$MSCs from bone marrow was rapidly upregulated in culture under normoxia to levels comparable to $\mathrm{CD}_{146}{ }^{+} \mathrm{LNGFR}^{+}$cells. The relationship between the CD146 ${ }^{+}$sorted cells in the study by $\mathrm{Wu}$ et al.[55*] and the cells marked by CD146 expression in vivo is therefore not clear.

An in-depth analysis of MSC lineages and subtypes in vivo will aid investigations aimed at addressing whether MSCs have a specific function to regulate immune homeostasis in their native tissues, and whether immunomodulation is a generic function of MSCs or specific to a distinct MSC subset. In a recent study[56*], clonal analysis of immortalised human BMMSCs revealed the existence of clones lacking multipotency that were positive for CD317 and were enriched for immunomodulatory transcriptional networks. Lineage tracing in mice allowed the identification of a rare non-differentiating BM-MSC subtype, distinct from perivascular MSCs, which was also found at 1\%-3\% frequency in human BM-MSC fractions[56*]. An intriguing scenario in RA pathogenesis is that stromal cells become unable to control the aberrant immune system and instead contribute to the perpetuation of joint inflammation in liaison with the immune system[57].

\section{MSCs and repair}

The repair potential of MSCs has been extensively studied preclinically and trialled in patients with joint surface defects and/or osteoarthritis with promising results (for review see[58]). In a recent proof-of-concept phase I/II clinical trial, the intra-articular injection of autologous Ad-MSCs into the osteoarthritic knee improved function and pain without causing adverse events, and reduced cartilage defects as determined by MRI, with histological evidence of hyaline cartilage repair[59]. Instead, the use of MSCs in RA has primarily focused on immune modulation, and the prevailing view is that MSCs prevent joint damage 
mainly via their immunosuppressive and anti-inflammatory activity. Evidence that the injected MSCs would contribute directly to joint tissue repair is scant. Bioluminescence imaging to trace luciferase-transfected MSCs after intra-articular injection in mice with proteoglycan-induced arthritis showed that MSCs were retained for several weeks in the injected joint[60], raising the possibility that injected MSCs could directly or indirectly modulate the local stromal compartment to promote intrinsic tissue repair. The recent identification of MSC populations in vivo and development of lineage tracing models will help address this knowledge gap.

An exciting prospect is the use of MSC-derived extracellular vesicles (EV), which have been shown to play roles in mediating tissue regeneration and immunomodulation[61*,62]. Intriguingly, the beneficial properties of EV may not be reliant on stem cells only. A recent study demonstrated that neutrophil-derived EV delivered into the knee joints of mice with serum-transfer arthritis displayed anti-inflammatory properties, prevented cartilage degradation, and favoured cartilage anabolism by penetrating the avascular cartilage extracellular matrix to deliver bioactive molecules to the chondrocytes[63**]. EV, directly or loaded with therapeutics, could thus be harnessed as a therapeutic strategy in RA.

\section{Clinical evaluation of MSC therapy in RA}

Intravenous infusion of allogeneic BM- or UC-MSCs into 4 patients with established RA, resistant to DMARDs and at least one anti-TNF $\alpha$ agent, was safe and resulted in partial and transient clinical improvement[64]. Intravenous injection of UC-MSCs in addition to DMARDs in 136 patients with active RA who had inadequate responses to traditional medication induced a significant clinical improvement when compared with the control group of 36 patients who received DMARDs plus medium without MSCs. The therapeutic effects were maintained for 3-6 months, and correlated with an increased percentage of Tregs in peripheral blood[65]. In a recent multicentre, dose-escalation, randomised, single-blind (double-blind for efficacy), placebo-controlled, phase Ib/IIa clinical trial[66**], intravenous infusions of allogeneic Ad-MSCs in 46 patients with active refractory RA (with failure to at least two biologics) were in general well-tolerated without evidence of toxicity over 3 months. There was a trend for clinical benefit, which was not persistent after 3 months, suggesting that cell therapy in RA would require repeated administration. However, in some 
patients sensitisation against allogeneic cells was detected[66**], calling for caution in multiple cell infusions.

In summary, preliminary data in human trials indicate that allogeneic MSCs could be effective in RA but larger, multicentre clinical studies are needed for sound evidence. So far, the use of MSCs in clinical studies has been restricted to patients with severe RA refractory to standard therapies. MSC treatment could be more effective if given at early stages of RA in order to "reset" the immune system by inducing regulatory networks. The selection criteria of RA patients for clinical studies will be crucial.

\section{Conclusions}

Advances have been made in understanding the mechanisms of the protective effects of MSC therapy in RA (Figure 12 ). The putative ability of MSCs to rewire an autoimmune process into a more naïve, tolerant state is an exciting concept. The study of the joint microenvironment and its interactions with the delivered cell populations will be crucial to maximise MSC therapeutic potential. The expanding knowledge of the mechanisms of MSC therapeutic effects will contribute to our understanding of the molecular taxonomy of RA, will inform patient stratification, and will unravel additional targets for pharmacological interventions in our journey to precision rheumatology. 


\section{Key points}

- Preclinical studies indicate that MSCs dampen joint inflammation via modulation of the adaptive and innate immune system.

- MSCs do not seem to contribute directly to tissue repair but their immunomodulatory effects prevent joint damage and may favour intrinsic repair.

- Clinical studies have shown a satisfactory safety profile of allogeneic MSCs in RA with promising trends for clinical efficacy but larger, multicentre clinical trials are needed. 


\section{Acknowledgements}

The authors thank all members of the Arthritis \& Regenerative Medicine Laboratory.

\section{Financial support}

The authors are grateful for support to their research from Arthritis Research UK (grants 19271, 19429, 19667, 20050, 20775) and the Medical Research Council (grant no. MR/L020211/1).

\section{Conflicts-of-Interest}

The authors have no conflict-of-interest to disclose. 


\section{REFERENCES:}

1. $\quad$ McInnes IB, Schett G. The pathogenesis of rheumatoid arthritis. N Engl J Med 2011; 365:2205-2219.

2. McInnes IB, Buckley CD, Isaacs JD. Cytokines in rheumatoid arthritis - shaping the immunological landscape. Nat Rev Rheumatol 2016; 12:63-68.

3. Dominici M, Le Blanc K, Mueller I, et al. Minimal criteria for defining multipotent mesenchymal stromal cells. The International Society for Cellular Therapy position statement. Cytotherapy 2006; 8:315-317.

4. Pittenger MF, Mackay AM, Beck SC, et al. Multilineage potential of adult human mesenchymal stem cells. Science 1999; 284 :143-147.

5. De Bari C, Dell’Accio F, Tylzanowski P, Luyten FP. Multipotent mesenchymal stem cells from adult human synovial membrane. Arthritis Rheum 2001; 44:1928-1942.

6. De Bari C, Dell'Accio F, Vandenabeele F, et al. Skeletal muscle repair by adult human mesenchymal stem cells from synovial membrane. J Cell Biol 2003; 160:909-918.

7. De Bari C, Dell'Accio F, Luyten FP. Human periosteum-derived cells maintain phenotypic stability and chondrogenic potential throughout expansion regardless of donor age. Arthritis Rheum 2001; 44:85-95.

8. De Bari C, Dell'Accio F, Vanlauwe J, et al. Mesenchymal multipotency of adult human periosteal cells demonstrated by single-cell lineage analysis. Arthritis Rheum 2006; 54:1209-1221.

9. Zuk PA, Zhu M, Ashjian P, et al. Human Adipose Tissue Is a Source of Multipotent Stem Cells. Mol Biol Cell 2002; 13:4279-4295.

10. Wang H-S, Hung S-C, Peng S-T, et al. Mesenchymal stem cells in the Wharton's jelly of the human umbilical cord. Stem Cells 2004; 22:1330-1337.

11. Uccelli A, de Rosbo NK. The immunomodulatory function of mesenchymal stem cells: mode of action and pathways. Ann N Y Acad Sci 2015; 1351:114-126.

12. Macdonald GI, Augello A, De Bari C. Mesenchymal stem cells: Re-establishing immunological tolerance in autoimmune rheumatic diseases. Arthritis Rheum 2011; 63:2547-2557. 
13. Augello A, Tasso R, Negrini SM, et al. Cell therapy using allogeneic bone marrow mesenchymal stem cells prevents tissue damage in collagen-induced arthritis. Arthritis Rheum 2007; 56:1175-1186.

14. González MA, Gonzalez-Rey E, Rico L, et al. Treatment of experimental arthritis by inducing immune tolerance with human adipose-derived mesenchymal stem cells. Arthritis Rheum 2009; 60:1006-1019.

15. Gonzalez-Rey E, Gonzalez M, Varela N, et al. Human adipose-derived mesenchymal stem cells reduce inflammatory and $\mathrm{T}$ cell responses and induce regulatory $\mathrm{T}$ cells in vitro in rheumatoid arthritis. Ann Rheum Dis 2010; 69:241-248.

* 16. Lopez-Santalla M, Mancheño-Corvo P, Menta R, et al. Human Adipose-Derived Mesenchymal Stem Cells Modulate Experimental Autoimmune Arthritis by Modifying Early Adaptive T Cell Responses. Stem Cells 2015; 33:3493-3503.

This study provides a detailed analysis of changes in $\mathrm{T}$ cell subset frequencies in spleen, peripheral blood, and draining lymph nodes in CIA mice after intravenous Ad-MSC administration.

17. Cornish AL, Campbell IK, McKenzie BS, et al. G-CSF and GM-CSF as therapeutic targets in rheumatoid arthritis. Nat Rev Rheumatol 2009; 5:554-559.

18. Ghannam S, Pène J, Moquet-Torcy G, et al. Mesenchymal stem cells inhibit human Th17 cell differentiation and function and induce a T regulatory cell phenotype. $\mathrm{J}$ Immunol 2010; 185:302-312.

19. Obermajer N, Popp FC, Soeder Y, et al. Conversion of Th17 into IL-17A(neg) regulatory $\mathrm{T}$ cells: a novel mechanism in prolonged allograft survival promoted by mesenchymal stem cell-supported minimized immunosuppressive therapy. J Immunol 2014; 193:4988-4999.

** 20. Luz-Crawford P, Tejedor G, Mausset-Bonnefont AL, et al. Glucocorticoid-induced leucine zipper governs the therapeutic potential of mesenchymal stem cells by inducing a switch from pathogenic to regulatory Th17 cells in a mouse model of collagen-induced arthritis. Arthritis Rheumatol 2015; 67:1514-1524.

This study shows that MSCs promote Th17 cells to adopt a regulatory phenotype, and demonstrates in vivo in CIA mice that this is dependent on expression by MSCs of 
glucocorticoid-induced leucine zipper, a modulator of NFкB and AP-1 signalling.

*21. Yan X, Cen Y, Wang Q. Mesenchymal stem cells alleviate experimental rheumatoid arthritis through microRNA-regulated IkappaB expression. Sci Rep 2016; 6:28915.

This paper implicates downregulation of microRNA (miR)-548e and a resulting de-repression of $\mathrm{I} \kappa \mathrm{B}$ translation and inhibition of $\mathrm{NF} \kappa \mathrm{B}$ signalling in the protective effects of intraperitoneal injection of syngeneic bone marrow MSCs in CIA mice.

22. Ma CS, Deenick EK. Human T follicular helper (Tfh) cells and disease. Immunol Cell Biol 2014; 92:64-71.

** 23. Liu R, Li X, Zhang Z, et al. Allogeneic mesenchymal stem cells inhibited T follicular helper cell generation in rheumatoid arthritis. Sci Rep 2015; 5:12777.

This paper reports inhibitory effects of human UC-MSCs on Follicular helper T (Tfh) cells in vitro and after systemic administration to CIA mice, which may mediate suppressive effects of MSCs on B cells.

*24. Rosado MM, Bernardo ME, Scarsella M, et al. Inhibition of B-cell proliferation and antibody production by mesenchymal stromal cells is mediated by T cells. Stem Cells Dev 2015; 24:93-103.

This study shows that the inhibitory effects of MSCs on B cells in vitro are largely mediated by effects on T cells, with a requirement for direct cell-cell interactions between MSCs and T cells, but not B cells.

*25. Lim JY, Im KI, Lee ES, et al. Enhanced immunoregulation of mesenchymal stem cells by IL-10-producing type 1 regulatory $\mathrm{T}$ cells in collagen-induced arthritis. Sci Rep 2016; 6:26851.

This study shows synergistic effects of co-administration of MSCs with Tr1 cells on arthritis in the CIA model, possibly through upregulation of IDO expression in MSCs induced by Tr1derived cytokines.

26. Ankrum JA, Dastidar RG, Ong JF, et al. Performance-enhanced mesenchymal stem cells via intracellular delivery of steroids. Sci Rep 2014; 4:4645.

27. Schett G, Teitelbaum SL. Osteoclasts and arthritis. J Bone Miner Res 2009; 24:11421146. 
28. Kelchtermans H, Geboes L, Mitera T, et al. Activated CD4+CD25+ regulatory T cells inhibit osteoclastogenesis and collagen-induced arthritis. Ann Rheum Dis 2009; 68:744-750.

29. Zaiss MM, Frey B, Hess A, et al. Regulatory T cells protect from local and systemic bone destruction in arthritis. J Immunol 2010; 184:7238-7246.

30. Oshita K, Yamaoka K, Udagawa N, et al. Human mesenchymal stem cells inhibit osteoclastogenesis through osteoprotegerin production. Arthritis Rheum 2011; 63:1658-1667.

31. Varin A, Pontikoglou C, Labat E, et al. CD200R/CD200 inhibits osteoclastogenesis: new mechanism of osteoclast control by mesenchymal stem cells in human. PLoS One 2013; 8:e72831.

*32. Garimella MG, Kour S, Piprode V, et al. Adipose-Derived Mesenchymal Stem Cells Prevent Systemic Bone Loss in Collagen-Induced Arthritis. J Immunol 2015; 195:5136-5148.

This study reports a protective effect of syngeneic Ad-MSCs on both local and systemic bone loss, associated with increased Tregs and Bregs and a decrease in osteoclast precursors in bone marrow.

33. Schurgers E, Kelchtermans H, Mitera T, et al. Discrepancy between the in vitro and in vivo effects of murine mesenchymal stem cells on T-cell proliferation and collageninduced arthritis. Arthritis Res Ther 2010; 12:R31.

34. Le Blanc K, Tammik C, Rosendahl K, et al. HLA expression and immunologic properties of differentiated and undifferentiated mesenchymal stem cells. Exp Hematol 2003; 31:890-896.

35. Zangi L, Margalit R, Reich-Zeliger S, et al. Direct imaging of immune rejection and memory induction by allogeneic mesenchymal stromal cells. Stem Cells 2009; 27:2865-2874.

36. Nauta AJ, Westerhuis G, Kruisselbrink AB, et al. Donor-derived mesenchymal stem cells are immunogenic in an allogeneic host and stimulate donor graft rejection in a nonmyeloablative setting. Blood 2006; 108:2114-2120.

37. Griffin MD, Ryan AE, Alagesan S, et al. Anti-donor immune responses elicited by 
allogeneic mesenchymal stem cells: what have we learned so far? Immunol Cell Biol 2013; 91:40-51.

38. Sakaguchi Y, Sekiya I, Yagishita K, Muneta T. Comparison of human stem cells derived from various mesenchymal tissues: Superiority of synovium as a cell source. Arthritis Rheum 2005; 52:2521-2529.

39. De Bari C, Dell’Accio F, Karystinou A, et al. A biomarker-based mathematical model to predict bone-forming potency of human synovial and periosteal mesenchymal stem cells. Arthritis Rheum 2008; 58:240-250.

40. Melief SM, Zwaginga JJ, Fibbe WE, Roelofs H. Adipose tissue-derived multipotent stromal cells have a higher immunomodulatory capacity than their bone marrowderived counterparts. Stem Cells Transl Med 2013; 2:455-463.

* 41. Gonzalo-Gil E, Perez-Lorenzo MJ, Galindo M, et al. Human embryonic stem cellderived mesenchymal stromal cells ameliorate collagen-induced arthritis by inducing host-derived indoleamine 2,3 dioxygenase. Arthritis Res Ther 2015; 18:77.

This study shows that intraperitoneal administration of MSCs derived from human embryonic stem cells can ameliorate CIA when administered after disease onset, and provides evidence for MSC homing to draining lymph nodes and upregulation of host-derived IDO expression.

42. Morikawa S, Mabuchi Y, Kubota Y, et al. Prospective identification, isolation, and systemic transplantation of multipotent mesenchymal stem cells in murine bone marrow. J Exp Med 2009; 206:2483-2496.

43. Zhou BO, Yue R, Murphy MM, et al. Leptin-receptor-expressing mesenchymal stromal cells represent the main source of bone formed by adult bone marrow. Cell Stem Cell 2014; 15:154-168.

44. Ding L, Saunders TL, Enikolopov G, Morrison SJ. Endothelial and perivascular cells maintain haematopoietic stem cells. Nature 2012; 481:457-462.

45. Méndez-Ferrer S, Michurina T V, Ferraro F, et al. Mesenchymal and haematopoietic stem cells form a unique bone marrow niche. Nature 2010; 466:829-834.

** 46. Worthley DL, Churchill M, Compton JT, et al. Gremlin 1 Identifies a Skeletal Stem Cell with Bone, Cartilage, and Reticular Stromal Potential. Cell 2015; 160:269-284. 
This paper identifies a novel population of osteochondroreticular stem cells in vivo in mouse bone marrow based on expression of Gremlin1 and shows these cells to contribute to bone formation during development and after femoral fracture.

47. Kurth TB, Dell'Accio F, Crouch V, et al. Functional mesenchymal stem cell niches in adult mouse knee joint synovium in vivo. Arthritis Rheum 2011; 63:1289-1300.

48. Jones EA, Kinsey SE, English A, et al. Isolation and characterization of bone marrow multipotential mesenchymal progenitor cells. Arthritis Rheum 2002; 46:3349-3360.

49. Quirici N, Soligo D, Bossolasco P, et al. Isolation of bone marrow mesenchymal stem cells by anti-nerve growth factor receptor antibodies. Exp Hematol 2002; 30:783-791.

50. Sacchetti B, Funari A, Michienzi S, et al. Self-Renewing Osteoprogenitors in Bone Marrow Sinusoids Can Organize a Hematopoietic Microenvironment. Cell 2007; $131: 324-336$.

51. Crisan M, Yap S, Casteilla L, et al. A perivascular origin for mesenchymal stem cells in multiple human organs. Cell Stem Cell 2008; 3:301-313.

52. Tormin A, Li O, Brune JC, et al. CD146 expression on primary nonhematopoietic bone marrow stem cells is correlated with in situ localization. Blood 2011; 117:50675077.

53. Pinho S, Lacombe J, Hanoun M, et al. PDGFR $\alpha$ and CD51 mark human nestin+ sphere-forming mesenchymal stem cells capable of hematopoietic progenitor cell expansion. J Exp Med 2013; 210:1351-1367.

54. Li H, Ghazanfari R, Zacharaki D, et al. Low/Negative Expression of PDGFR- $\alpha$ Identifies the Candidate Primary Mesenchymal Stromal Cells in Adult Human Bone Marrow. Stem Cell Reports 2014; 3:965-974.

*55. Wu CC, Liu FL, Sytwu HK, et al. CD146+ mesenchymal stem cells display greater therapeutic potential than CD146- cells for treating collagen-induced arthritis in mice. Stem cell Res Ther 2016; 7:23.

This study shows that sorting of culture-expanded human UC-MSCs for CD146 expression enriches for cells with immunosuppressive properties with efficacy in CIA mice.

*56. James S, Fox J, Afsari F, et al. Multiparameter Analysis of Human Bone Marrow 
Stromal Cells Identifies Distinct Immunomodulatory and Differentiation-Competent Subtypes. Stem Cell Reports 2015; 4:1004-1015.

This study identifies, through clonal analysis of immortalised human BM-MSCs, a rare nondifferentiating BM-MSC subset, marked by CD317 expression and high IL-7 production, with significantly enriched immunomodulatory transcriptional networks, which was also present in human BM-MSCs.

57. De Bari C. Are mesenchymal stem cells in rheumatoid arthritis the good or bad guys? Arthritis Res Ther 2015; 17:113.

58. Roelofs AJ, Rocke JP, De Bari C. Cell-based approaches to joint surface repair: a research perspective. Osteoarthritis Cartilage 2013; 21:892-900.

59. Jo CH, Lee YG, Shin WH, et al. Intra-Articular Injection of Mesenchymal Stem Cells for the Treatment of Osteoarthritis of the Knee: A Proof-of-Concept Clinical Trial. Stem Cells 2014; 32:1254-1266.

60. Swart JF, de Roock S, Hofhuis FM, et al. Mesenchymal stem cell therapy in proteoglycan induced arthritis. Ann Rheum Dis 2015; 74:769-777.

*61. Zhang S, Chu WC, Lai RC, et al. Exosomes derived from human embryonic mesenchymal stem cells promote osteochondral regeneration. Osteoarthr Cartil 2016. doi:10.1016/j.joca.2016.06.022.

This study shows that weekly intra-articular injections of human embryonic MSC-derived exosomes in a rat osteochondral defect model stimulated repair of the joint surface.

62. Blazquez R, Sanchez-Margallo FM, de la Rosa O, et al. Immunomodulatory Potential of Human Adipose Mesenchymal Stem Cells Derived Exosomes on in vitro Stimulated T Cells. Front Immunol 2014; 5:556.

**63. Headland SE, Jones HR, Norling L V, et al. Neutrophil-derived microvesicles enter cartilage and protect the joint in inflammatory arthritis. Sci Transl Med 2015; 7:315.

This study demonstrated that intra-articular administration of neutrophil-derived EV in the serum transfer model of inflammatory arthritis displayed anti-inflammatory properties, prevented cartilage degradation, and favoured cartilage anabolism by penetrating the avascular cartilage extracellular matrix to deliver bioactive molecules to the chondrocytes. 
64. Liang J, Li X, Zhang $\mathrm{H}$, et al. Allogeneic mesenchymal stem cells transplantation in patients with refractory RA. Clin Rheumatol 2012; 31:157-161.

65. Wang L, Wang L, Cong X, et al. Human umbilical cord mesenchymal stem cell therapy for patients with active rheumatoid arthritis: safety and efficacy. Stem Cells Dev 2013; 22:3192-3202.

**66. Álvaro-Gracia JM, Jover JA, García-Vicuña R, et al. Intravenous administration of expanded allogeneic adipose-derived mesenchymal stem cells in refractory rheumatoid arthritis (Cx611): results of a multicentre, dose escalation, randomised, single-blind, placebo-controlled phase Ib/IIa clinical trial. Ann Rheum Dis 2016. doi:10.1136/annrheumdis-2015-208918.

This multicentre, dose-escalation clinical trial of intravenous infusions of allogeneic AdMSCs in patients with active refractory RA showed therapy was generally well-tolerated, with a trend for clinical efficacy warranting further investigation. 


\section{Figure 1: Sources of MSCs discussed in this review.}

Figure 12: Schematic of proposed mechanisms of MSC therapy in RA. Mesenchymal Stem Cell (MSC), Interleukin 10 (IL-10), Prostaglandin E2 (PGE-2), Indoleamine 2,3dioxygenase (IDO), Nitric oxide (NO), Interferon gamma (INF-y), Immature Dendritic Cell (iDM), Mature Dendritic Cell (mDC), Pro-inflammatory macrophage (M1), Antiinflammatory Macrophage (M2). 


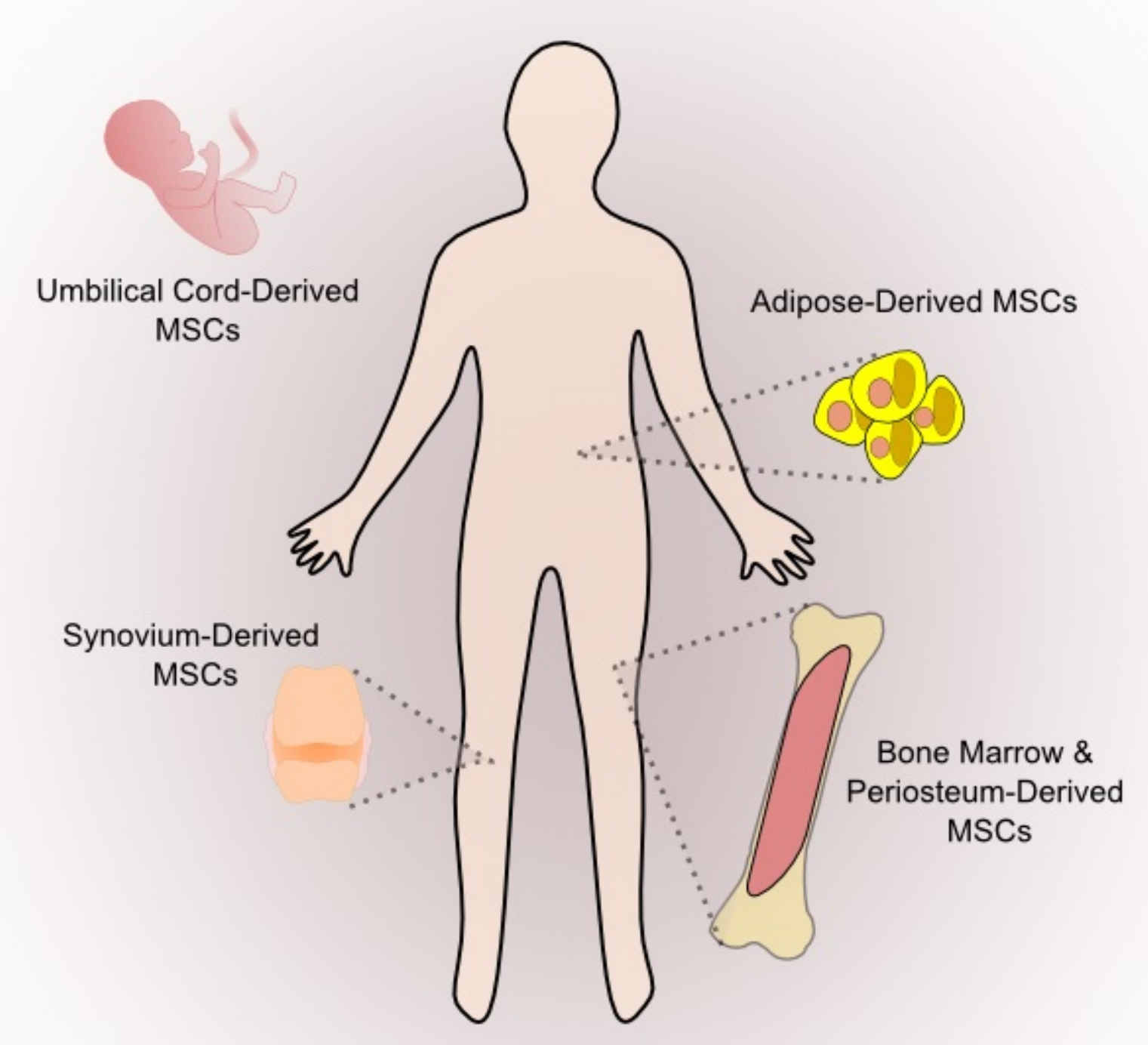




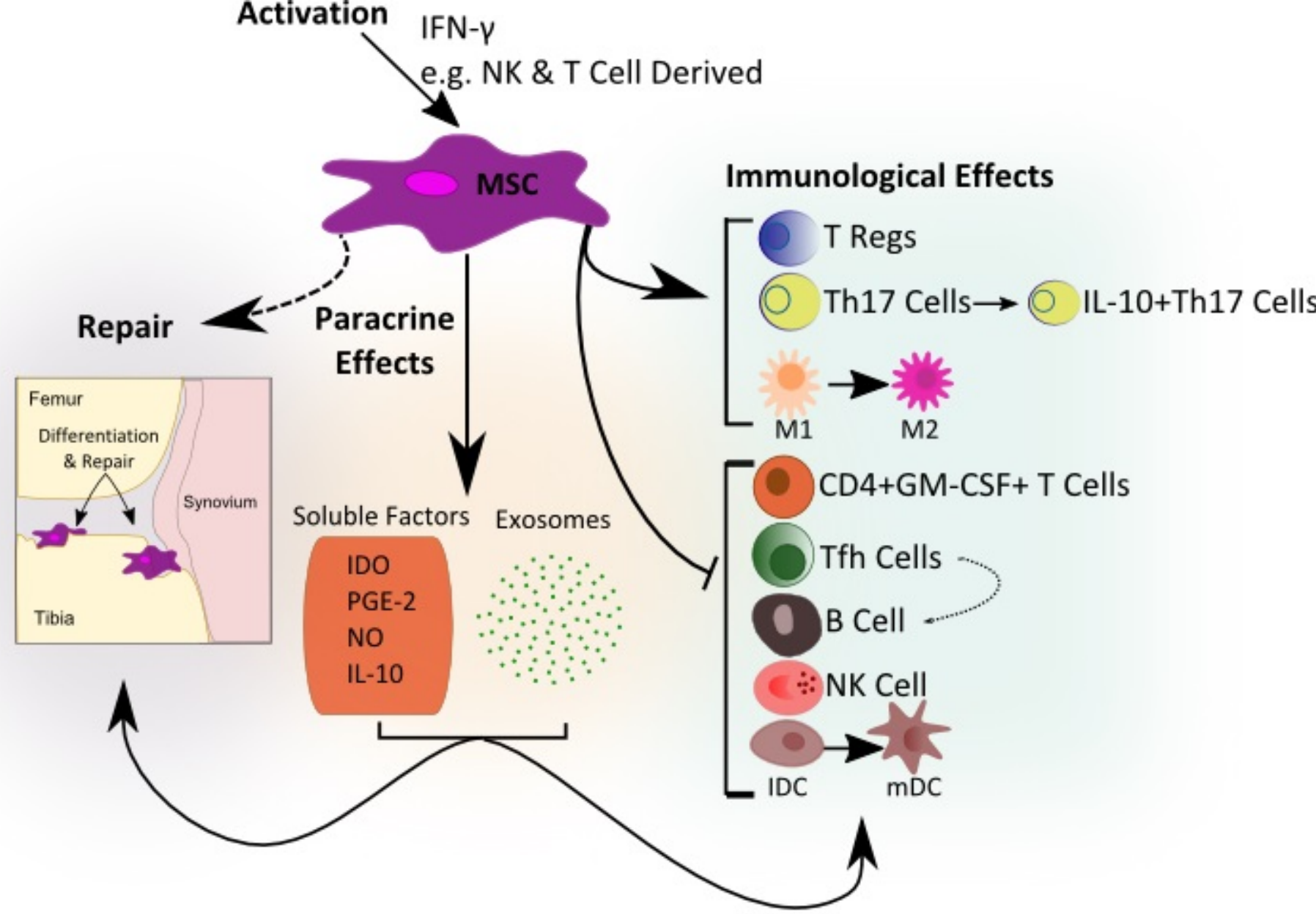

